\title{
Comment on 'Fixed point theorems for contraction mappings in modular metric spaces, Fixed Point Theory and Applications, doi:10.1186/1687-1812-2011-93, 20 pages'
}

H Dehghan' ${ }^{1}$ M Eshaghi Gordji2 ${ }^{*}$ and A Ebadian ${ }^{3 *}$

\section{"Correspondence:}

meshaghi@semnan.ac.ir; ebadian.ali@gmail.com

${ }^{2}$ Department of Mathematics, Semnan University, P.O. Box

35195-363, Semnan, Iran

${ }^{3}$ Department of Mathematics,

Payame Noor University, Tehran, Iran

Full list of author information is

available at the end of the article

\begin{abstract}
In this paper, we provide an example to show that some results obtained in [Mongkolkeha et al. in Fixed Point Theory Appl. 2011, doi:10.1186/1687-1812-2011-93] are not valid.

MSC: $47 \mathrm{H} 09 ; 47 \mathrm{H} 10$
\end{abstract}

Keywords: contraction mappings; modular metric spaces; metric space

Definition 1 [1] Let $X$ be a nonempty set. A function $\omega:(0, \infty) \times X \times X \rightarrow[0, \infty]$ is said to be metric modular on $X$ if for all $x, y, z \in X$, the following conditions hold:

(i) $\omega_{\lambda}(x, y)=0$ for all $\lambda>0$ iff $x=y$;

(ii) $\omega_{\lambda}(x, y)=\omega_{\lambda}(y, x)$ for all $\lambda>0$;

(iii) $\omega_{\lambda+\mu}(x, y) \leq \omega_{\lambda}(x, z)+\omega_{\mu}(z, y)$ for all $\lambda, \mu>0$.

Given $x_{\star} \in X$, the set $X_{\omega}\left(x_{\star}\right)=\left\{x \in X: \lim _{\lambda \rightarrow \infty} \omega_{\lambda}\left(x, x_{\star}\right)=0\right\}$ is called a modular metric space generated by $x_{\star}$ and induced by $\omega$. If its generator $x_{\star}$ does not play any role in the situation, we will write $X_{\omega}$ instead of $X_{\omega}\left(x_{\star}\right)$.

We need the following theorems in the proof of the main result of this paper.

Theorem 2 [1, Theorem 2.6] If $\omega$ is metric (pseudo) modular on $X$, then the modular set $X_{\omega}$ is a (pseudo) metric space with (pseudo) metric given by

$$
d_{\omega}^{\circ}(x, y)=\inf \left\{\lambda>0: \omega_{\lambda}(x, y) \leq \lambda\right\}, \quad x, y \in X_{\omega} .
$$

Theorem 3 [1, Theorem 2.13] Let $\omega$ be (pseudo) modular on a set X. Given a sequence $\left\{x_{n}\right\} \subset X_{\omega}$ and $x \in X_{\omega}$, we have $d_{\omega}^{\circ}\left(x_{n}, x\right) \rightarrow 0$ as $n \rightarrow \infty$ if and only if $\omega_{\lambda}\left(x_{n}, x\right) \rightarrow 0$ as $n \rightarrow \infty$ for all $\lambda>0$. A similar assertion holds for Cauchy sequences.

(C) 2012 Dehghan et al.; licensee Springer. This is an Open Access article distributed under the terms of the Creative Commons Attribution License (http://creativecommons.org/licenses/by/2.0), which permits unrestricted use, distribution, and reproduction in any medium, provided the original work is properly cited. 
Let $\omega$ be modular on a set $X$. A mapping $T: X_{\omega} \rightarrow X_{\omega}$ is said to be contraction [2, Definition 3.1] if there exists $k \in[0,1)$ such that

$$
\omega_{\lambda}(T x, T y) \leq k \omega_{\lambda}(x, y)
$$

for all $\lambda>0$ and $x, y \in X_{\omega}$.

Recently, Mongkolkeha et al. [2] proved the following theorems.

Theorem 4 [2, Theorem 3.2] Let $\omega$ be metric modular on $X$ and $X_{\omega}$ be a modular metric space induced by $\omega$. If $X_{\omega}$ is a complete modular metric space and $T: X_{\omega} \rightarrow X_{\omega}$ is a contraction mapping, then $T$ has a unique fixed point in $X_{\omega}$. Moreover, for any $x \in X_{\omega}$, iterative sequence $\left\{T^{n}(x)\right\}$ converges to the fixed point.

Theorem 5 [2, Theorem 3.4] Let $\omega$ be metric modular on $X$ and $X_{\omega}$ be a modular metric space induced by $\omega$. If $X_{\omega}$ is a complete modular metric space and $T: X_{\omega} \rightarrow X_{\omega}$ is a mapping, which $T^{N}$ is a contraction mapping for some positive integer $N$. Then, $T$ has a unique fixed point in $X_{\omega}$.

We show that Theorems 4 and 5 are not correct. To this end, we give the following example.

Example 6 Let $X=\mathbb{R}$ and define modular $\omega$ by $\omega_{\lambda}(x, y)=\infty$ if $\lambda \leq|x-y|$, and $\omega_{\lambda}(x, y)=0$ if $\lambda>|x-y|$. It is easy to verify that (see also [1, Example 2.7]) $X_{\omega}=\mathbb{R}$ and $d_{\omega}^{\circ}(x, y)=|x-y|$. It follows from Theorem 3 that $\mathbb{R}$ is a complete modular metric space. Now, define $T$ : $\mathbb{R} \rightarrow \mathbb{R}$ by $T x=x+1$. We show that $T$ is a contraction while it has no fixed point. Let $k \in[0,1)$ (for example, $k=1 / 2$ ) and $x, y \in \mathbb{R}$. If $\lambda \leq|x-y|$, then $\omega_{\lambda}(x, y)=\infty$ and (1) holds. If $|x-y|<\lambda$, then $|T x-T y|=|x-y|<\lambda$. Therefore, $\omega_{\lambda}(T x, T y)=\omega_{\lambda}(x, y)=0$. Hence $T$ is a contraction. On the other hand, by definition of $T$, it is easy to see that $T$ has no fixed point. So, Theorems 4 and 5 are not correct.

Remark 7 In [2, Example 3.7], the authors mentioned that 'Thus, $T$ is not a contraction mapping and then the Banach contraction mapping cannot be applied to this example.' It is true that $T$ is not contraction with the Euclidean metric, but one can easily verify that

$$
d_{\omega}^{\circ}(T x, T y) \leq \frac{\sqrt{3}}{2} d_{\omega}^{\circ}(x, y)
$$

Thus, the Banach contraction guarantees the existence of a fixed point. Note that

$$
d_{\omega}^{\circ}\left(\left(a_{1}, 0\right),\left(a_{2}, 0\right)\right)=\sqrt{\frac{4\left|a_{1}-a_{2}\right|}{3}}, \quad d_{\omega}^{\circ}\left(\left(0, b_{1}\right),\left(0, b_{2}\right)\right)=\sqrt{\left|b_{1}-b_{2}\right|}
$$

and

$$
d_{\omega}^{\circ}((a, 0),(0, b))=\sqrt{\frac{4 a}{3}+b} .
$$


Competing interests

The authors declare that they have no competing interests.

\section{Authors' contributions}

All authors conceived of the study, participated in its design and coordination, drafted the manuscript, participated in the sequence alignment, and read and approved the final manuscript.

\section{Author details}

${ }^{1}$ Department of Mathematics, Institute for Advanced Studies in Basic Sciences (IASBS), Gava Zang, Zanjan, 45137-66731, Iran. ${ }^{2}$ Department of Mathematics, Semnan University, P.O. Box 35195-363, Semnan, Iran. ${ }^{3}$ Department of Mathematics, Payame Noor University, Tehran, Iran.

Received: 8 January 2012 Accepted: 22 August 2012 Published: 7 September 2012

\section{References}

1. Chistyakov, WV: Modular metric spaces, I: basic concepts. Nonlinear Anal. 72, 1-14 (2010)

2. Mongkolkeha, C, Sintunavarat, W, Kumam, P: Fixed point theorems for contraction mappings in modular metric spaces. Fixed Point Theory Appl. (2011). doi:10.1186/1687-1812-2011-93

doi:10.1186/1687-1812-2012-144

Cite this article as: Dehghan et al.: Comment on 'Fixed point theorems for contraction mappings in modular metric spaces, Fixed Point Theory and Applications, doi:10.1186/1687-1812-2011-93, 20 pages'. Fixed Point Theory and Applications 2012 2012:144.

\section{Submit your manuscript to a SpringerOpen ${ }^{\circ}$ journal and benefit from:}

- Convenient online submission

- Rigorous peer review

Immediate publication on acceptance

- Open access: articles freely available online

- High visibility within the field

- Retaining the copyright to your article 